\title{
Arbor
}

\section{El Mediterráneo, más cerca y más lejos}

Xavier Vidal-Folch

Arbor CLXXII, 678 (Junio 2002), 383-406 pp.

\section{Iniciativa española}

Europa redescubrió el Mediterráneo en 1995, en la Conferencia de Barcelona. Lanzó un ambicioso proyecto de "región euromediterránea» articulada entre los Quince del Norte y doce países de la ribera del Sur ${ }^{1}$. Económicamente, debía desembocar en una zona de libre cambio para el año 2010; políticamente, en un paraíso de paz, seguridad, democracia, derechos humanos; socialmente, aproximaría los niveles de bienestar y cultura de ambas riberas.

El interés por el área no era nuevo. Desde los años sesenta, la Europa comunitaria había ido firmando una serie de acuerdos bilaterales y protocolos financieros, diseñados según parámetros de cooperación clásica, con los países ribereños extracomunitarios. Con ocasión de la guerra del golfo y tras la caída del muro de Berlín, se dio un salto cualitativo. Esos acuerdos se incardinaron en una "política mediterránea renovada», por iniciativa del comisario europeo Abel Matutes. Esta ampliaba el alcance cuantitativo de las iniciativas anteriores, al duplicar para el periodo 1992-1996 las cifras de cooperación al desarrollo hasta entonces ofrecidas. Y sobre todo, al incorporar una dimensión regional, puesto que estableció los programas Med (Urbs, entre ciudades; Campus, entre universidades; Invest, entre centros de investigación; y Media, entre medios de comunicación...), que beneficiaban simultáneamente, según reglas comunes, a los distintos vecinos.

Tampoco era nuevo el impulso español del nuevo proyecto euromediterráneo, de la mano del comisario Manuel Marín ${ }^{2}$, aunque se conoce menos que los padres de la idea de una zona mediterránea de libre cambio habían sido, en 1991, los marroquíes ${ }^{3}$. Las cumbres de Lisboa, Corfú y Es- 
sen sirvieron para evidenciar la necesidad de un paralelismo entre la aproximación al Sur respecto a la del Este. Los datos utilizados por los europeos mediterráneos para convencer de su necesidad e interés a sus socios norteños apelaban a que la ribera sur suponía un mercado de $304 \mathrm{mi}-$ llones de consumidores (frente a 116 del Este); un PIB de más de 240 millones de euros (frente a 150 millones del Este); un superávit comercial doble; una dependencia del Sur del $24 \%$ (frente al $1 \%$ ) y una inmigración de 4,6 millones de ciudadanos surmediterráneos (contra 700.000).

La continuidad del impulso político español daba cuenta de un doble fenómeno: la aportación de España al patrimonio comunitario (dimensiones mediterránea y latinoamericana), y la evidencia de que una política exterior de un Estado miembro de la Unión sólo alcanza masa crítica operativa si es absorbida por el conjunto, como política exterior de la Unión. Por alumbrar el proyecto y su dotación pugnaron, junto a Marín, Jacques Delors y Felipe González, en tensa connivencia presupuestaria con Helmut Kohl durante la cumbre de Cannes (junio de 1995), que acabó estableciendo un vínculo matemático (de 70 a 30) para las ayudas directas -esto es, aparte de los créditos del BEI- al Este europeo (6.692 millones de euros) y al Sur norteafricano (4.685 millones) para los siguientes cinco años. Eso suponía una relación Sur-Este de 1 a 1,14, mientras que entre 1991 a 1994 fue de 1 a 2,5 veces. Javier Solana, en su calidad de miembro de la presidencia de turno de la Unión Europea (UE), acabó logrando que cristalizase ese paralelismo con la estrategia de aproximación ya enhebrada para el Este europeo, al consagrar en la cumbre de Barcelona (27 y 28 de noviembre de 1995) un tratamiento de región a región.

La superioridad teórica de la arquitectura de la Euromed respecto a otras regiones económicas en ciernes era abrumadora. En comparación con la más reciente, la fraguada por el Tratado de Libre Comercio para América del Norte (TLC, entre EE UU, Canadá y México de 1994) estribaba no sólo en el amplio abanico de países destinatarios, sino también en su articulación sobre un triple pilar, político y de seguridad, económico, y humano-social, en vez del económico en solitario.

Más aún. En el capítulo económico, el proceso de Barcelona incorporaba la filosofía de la solidaridad o cohesión social y territorial propia de la UE, aunque con menor intensidad. Aportaba al Sur, en efecto, notables transferencias presupuestarias entendidas como contrapartidas a los costes de transición al mercado, como compensaciones a los sacrificios sociales que entraña el inicio de la liberalización, y como catalizadores del desarrollo endógeno de los vecinos y estabilizadores de su frágil estructura social en momentos de cambio. Si el modelo anglosajón del TLC 
El Mediterráneo, más cerca y más lejos

(como el de la fenecida EFTA) tomaba cuerpo sobre la idea «trade, not aid», el proyecto europeo cabalgaba sobre el doble lomo «del trade and aid». Una ilustración, al cabo, de que las políticas exteriores vienen a cristalizar hacia fuera los modelos internos.

Además, el mecanismo de asociación inventado exhibe bastante sofisticación. Por un lado es bilateral, vincula a cada uno de los doce sureños con la Unión, mediante tratados de «asociación euromediterránea» individualmente negociados; por otro, es relativamente multilateral, porque aunque esos tratados incluyen cláusulas específicas nacionales, responden a una plantilla común, incorporan un mismo sistema armonizador de normas técnicas, sanitarias y comerciales que debieran abocar a un desarme arancelario con la UE bastante simultáneo. Es vertical, porque enlaza Norte y Sur, pero también horizontal, porque posibilita acuerdos Sur/Sur que no contradigan el diseño global. Plantea objetivos comunes -como la zona de libre comercio para 2010- pero no impide velocidades distintas. Resulta así un marco bastante flexible -aunque complejoadaptable a países muy diferenciados y a situaciones multiformes.

\section{Frecuencia diplomática}

Pero entre el plano del arquitecto y la realidad del edificio media un trecho. A lo largo de toda su historia, el proceso de construcción euromediterránea se ha visto contaminado, incluso secuestrado, por el conflicto del Oriente Próximo, más concretamente entre Israel y Palestina.

Ni una sola de las conferencias-cumbre de ministros de Asuntos Exteriores (ha habido otras muchas de ministros sectoriales), primero a ritmo bianual y desde 2000 con frecuencia anual, ha logrado zafarse del conflicto. Así, la reunión fundacional de Barcelona (27 y 28 de noviembre de 1995) bordeó el fracaso. Los sirios querían añadir al párrafo que reconocía, enunciativamente, el derecho de autodeterminación de los pueblos, el «derecho de los pueblos bajo dominación ajena, colonial u otras», a «emprender acciones legítimas» contra ella; a Israel le disgustaba la referencia al respeto a la «integridad territorial de los Estados», por la cuestión del Líbano-sur y se enfrentaba a Egipto eludiendo compromisos de no proliferación nuclear si no se extendían a las -ausentes- Irán e Irak... Un órdago de la presidencia, lanzado en la madrugada de la última jornada, salvó in extremis la conferencia, y la carta magna del proceso o Declaración de Barcelona.

La conferencia de Malta (15 y 16 de abril de 1997) se celebró con malos augurios, el mandato duro de Benjamin Netanyahu había reeempla- 
zado en junio de 1996 al flexible de Simón Peres y la Liga Arabe acababa de decretar un boicoteo comercial y diplomático a Tel Aviv. En esas desfavorables circunstancias lo mejor del encuentro fue su propia realización -prolongada en un contacto bilateral entre el rais palestino, Yasir Arafat, y el ministro de Exteriores israelí David Levy- que, sin embargo, se clausuró sin redactarse siquiera una declaración final. La tercera conferencia, en Stuttgart (16 y 17 de abril de 1999), concluyó consagrando el borrador, aplazado desde Malta, de una Carta para la Paz y Estabilidad en la región, pero sólo a título de «documento de trabajo». Hubo conclusiones $-\mathrm{y}$ la inédita presencia de un representante libio con estatuto de observador-, pero no hubo conferencia de prensa de clausura, por la pretensión del ministro israelí, Ariel Sharon, de coprotagonizarla para equilibrar la presencia de su colega sirio, contra lo oficialmente previsto.

La cuarta convocatoria, en Marsella el 15 y 16 de noviembre de 2000, (que representaría el relanzamiento del proyecto, como veremos) venía prologada por una nueva fase de violencia en los territorios palestinos (200 muertos en dos meses), lo que fue utilizado por Siria y su satélite Líbano como coartada para negarse a asistir. Los ministros israelí y palestino -esta vez Shlomo Ben Ami y Nabil Shaath- protagonizaron algún rifirrafe dialéctico, pero esta vez las conclusiones fueron más prácticas. La quinta conferencia se celebró en Bruselas el 5 y 6 de noviembre de 2001, en plena intifada, y con varias ciudades palestinas ocupadas por tanques isarelíes en venganza por el asesinato del ministro de Turismo, Rehavam Zeevi. Lógicamente se volvió a registrar alta tensión cuando el isrrelí Simón Peres acusó a Damasco de dificultar el proceso de paz dando cobijo a terroristas y el sirio Faruk al Shara le llamó mentiroso y distinguió entre combatientes por la libertad y terroristas. Pero esta vez no hubo deserciones.

La convocatoria de la sexta conferencia (Valencia, 22 y 23 de abril de 2002) llegó con el momento más caliente del conflicto, que produjo centenares de muertes, la ocupación y destrucción de ciudades palestinas y el asedio israelí a la sede oficial de la Autoridad Palestina, en Ramala. Peor augurio, imposible.

Sirva este descriptor detallado para constatar la continua interferencia del drama de Oriente Medio en la vertebración de la región. Los más optimistas suelen destacar que -con vaivenes de distinto signo- esa asechanza no ha impedido erigir el foro euromediterráneo en el único escenario multilateral donde se reúnen todos los protagonistas de la zona, incluso los más enfrentados. Pero ello no es óbice para subrayar las consecuencias de ralentización, generación de recelos y establecimiento de obstáculos que ha supuesto para el proceso euromediterráneo. 
El Mediterráneo, más cerca y más lejos

Sin embargo, el panorama diplomático y técnico que se ha ido tejiendo quedaría incompleto sin mencionar algunas conferencias de ministros sectoriales como la del agua, en Turín, que diseñó algunos programas multilaterales (1999); o la del medio ambiente, en Helsinki (1997), que lanzó el programa de saneamiento SMAP, y sirvió para interesar más a los socios escandinavos de la UE en el Euromed. O un documento importante, la comunicación de la Comisión Europea «Sobre la Asociación Euromediterránea y el Mercado Único» ${ }^{4}$, que precisaba el horizonte de la zona de libre cambio prevista por la Declaración de Barcelona desde la experiencia de que el mercado único «es mucho más que una zona de libre comercio» y esbozaba un programa para suprimir las barreras al comercio en ámbitos como las aduanas, la fiscalidad, la contratación pública, la competencia y los derechos de propiedad intelectual.

También fue destacable la reunión de Agadir (7 de mayo de 2001) en la que los Ministros de Exteriores de Marruecos, Egipto, Túnez y Jordania firmaron un acuerdo de libre cambio abierto a otros socios del Sur. Aunque -contra lo comprometido- no concretó sus resultados durante el año de su celebración, abrió la espita a la expectativa de una aceleración de las relaciones horizontales Sur-Sur ${ }^{5}$.

\section{Magros resultados}

Pese a su contaminación a cuenta del conflicto del Próximo Oriente, el proceso euromediterráneo se mantuvo vivo. Aunque cinco años es un suspiro en la dinámica comunitaria -que suele registrar arranques muy lentos, frecuentemente generadores de una aceleración posterior-, no lo son tanto en la vida de los pueblos. Por eso, al doblar el cabo del primer quinquenio, se multiplicaron los análisis, reflexiones y debates sobre el balance y los resultados obtenidos. $\mathrm{Y}$ todos coincidieron en evaluarlos como muy mediocres.

\section{Lentitud de los acuerdos bilaterales}

El proceso de negociación y ratificación de los Acuerdos de Asociación fue «más lento de lo esperado» ${ }^{6}$, tanto por culpa del Norte, especialmente en la discusión de los ritmos de liberalización agrícola -como se analizará más adelante- y por la complejidad de los procedimientos parlamentarios nacionales, cuanto por las reticencias de algunos sureños a «acelerar el ritmo de la transición económica» y de efectuar las reformas 
exigidas por la asociación», así como por la gravedad de algunos de sus déficit democráticos.

De manera que de los nueve países con que se debían culminar esos acuerdos -pues de los doce, Chipre, Malta y Turquía ya tenían tratados en vigor que contemplan uniones aduaneras-, cinco años después de Barcelona menos de la mitad estaban en vigor: Túnez, Marruecos, Israel y la Autoridad Palestina. El de Jordania (1997) esperaba la ratificación; el de Egipto se había negociado y esperaba la firma, y los de Líbano, Siria y Argelia seguían en la mesa de discusión.

Esa lentitud gravaba todo el proceso de constitución en región, porque el objetivo de libre comercio multilateral no puede alcanzarse hasta que no rijan todos los acuerdos bilaterales, piezas de aquél. Dificultaba en concreto el avance hacia la liberalización comercial multilateral mediante la «acumulación diagonal de origen», a través de la cual un producto que incorpora elementos procedentes de varios países socios goza de las preferencias comerciales previstas. E impedía ulteriores pasos en la conversión de una zona de libre cambio en un área articulada con normas económicas e industriales armonizadas que faciliten la atracción de capitales, de modo que un inversor pueda desarrollar planes regionales y no país por país, lo que le genera costes adicionales, más aún tratándose de mercados de dimensión modesta.

\section{Seguridad y dimensión humana}

El objetivo estrella, la elaboración de una Carta para la Paz y la Estabilidad en la región que desarrollase los objetivos de seguridad consensuados en Barcelona, obtuvo un notable impulso en la cumbre de Stuttgart de 1999, donde los ministros de Exteriores de los 27 acordaron las cuestiones de principio para la elaboración de un texto. El obstáculo de Oriente Próximo implicó que, no sólo en los primeros cinco años sino hasta el momento presente, haya resultado extemporánea la redacción de esta Carta.

Como mal menor, se mantuvieron las reuniones ad-hoc de altos funcionarios, en la perspectiva de transformarlas en un foro institucional de diálogo sobre cuestiones como el terrorismo, la prevención de conflictos y la gestión de crisis.

La cooperación multilateral empezó a plasmarse en distintos programas relacionados con el agua y el medio ambiente, la sociedad de la información, la herencia cultural y las infrastructuras, aunque todos ellos de alcance relativamente modesto y visibilidad limitada. Y de los foros ci- 


\section{El Mediterráneo, más cerca y más lejos}

viles que acompañaron a las distintas cumbres puede concluirse lo mismo.

\section{Cooperación económica}

La relación económica existente en el punto de partida entre las dos riberas era el acentuado desequilibrio típico de la relación Norte-Sur. Y poco ha cambiado.

El progreso más destacado se detecta en el cuadro macroeconómico global, más que en la economía real. Los países del Sur avanzaron en el saneamiento de sus cuentas públicas y en la estabilización de sus principales variables macroeconómicas, merced a sus -tímidas y desigualesreformas económicas y al apoyo europeo a las mismas, dotado con $600 \mathrm{mi}$ llones de euros comprometidos, -que no significa ejecutados- (casi el 20\% del programa Meda) para Marruecos, Argelia, Túnez, Jordania y Líbano. Los resultados de esta política se recogen en el cuadro número 1:

Pero la diferencia de riqueza relativa, medida en paridades de poder de compra ajustadas -el baremo más exacto y a la vez menos escandaloso- era abismal, establecía una relación de cinco a uno entre Norte y Sur del Mediterráneo. Los dos instrumentos contemplados para combatir ese desequilibrio, comercio e inversión, arrojaron resultados menores.

Comercialmente, en 1995 las exportaciones del Sur al Norte totalizaban 33.398 millones de euros por 50.651 millones de euros en sentido inverso. El déficit del Sur era menos acusado en el ámbito agrícola en términos absolutos (3.553 millones de euros contra 4.394 millones) ${ }^{7}$, pero también el más grave en términos relativos, dado el peso del sector primario en las economías norteafricanas. Este panorama no ha cambiado sustancialmente. Tampoco se modificó la relación horizontal comercial

CUADRo 1. Variables macroeconómicas del sur

\begin{tabular}{||l|c|c|}
\hline & $\begin{array}{c}\text { Tasa de crecimiento } \\
\text { medio 1990-95 }\end{array}$ & $\begin{array}{c}\text { Tasa de crecimiento } \\
\text { medio 1996-1999 }\end{array}$ \\
\hline Tasa de inflación (\%) & $13,6 \%$ & $4,0 \%$ \\
Déficit presupuestario (en \% del PIB) & $5,2 \%$ & $3,2 \%$ \\
Tipo de interés real (en \%) & $2,0 \%$ & $5,2 \%$ \\
PIB (en \%) & $3,5 \%$ & $4,1 \%$ \\
\hline
\end{tabular}

Los datos incluyen a los 12 ribereños del Sur.

Fuente: Banco Mundial. 
Sur-Sur: el comercio entre los vecinos del Magreb y del Mashraq apenas alcanzaba el 5\% de sus transacciones exteriores; los mercados árabes permanecían y permanecen cerrados entre sí, desincentivando así la afluencia de inversión extranjera.

La inversión -privada y pública- tampoco desencalló el escaso desarrollo del Sur. El punto de partida era bajo. Según cálculo del experto Bichara Khader, profesor en Lovaina ${ }^{8}$, las inversiones europeas eran «ocho veces menores que las norteamericanas en México». El punto de llegada siguió siendo bajo: del total de inversiones directas de la UE en el conjunto de los países de economía emergente, la zona mediterránea absorbía a final de los noventa sólo el 5\%. Una ilustración -aunque en espacio temporal distinto-: en los últimos diez años las empresas españolas han invertido más de 60.000 millones de euros (diez billones de pesetas) en Argentina, por menos 3.000 millones de euros (medio billón de pesetas) en Marruecos.

Coherentemente, las inversiones privadas mundiales en la ribera sur no sólo no aumentaron en términos relativos, sino que decrecieron medio punto entre 1995 y 1998 respecto al quinquenio anterior, situándose por debajo del $1 \%$ del total mundial, como indica el cuadro número 2. Esa cifra de carácter residual contrastaba con el hecho de que el peso del área supone aproximadamente el $4 \%$ del comercio mundial y casi el $4 \%$ de la población . Expresado de otra manera, la ya escasa capacidad de atraer capitales disminuyó seriamente, casi un $40 \%$, mientras aumentaban las de Latinoamérica y Europa central y Oriental.

Es decir, mientras la inversión en Latinoamérica multiplicaba la realizada en el Sur mediterráneo por casi nueve veces $(8,75)$ a mitad de decenio, a finales de los noventa se multiplicaba por 17,4 veces. Y mientras la inversión en Europa central y oriental duplicaba la mediterránea a mitad de decenio, la quintuplicaba a final de decenio. Nótese que ambas constituyen zonas prioritarias de expansión de la UE.

La inversión pública no pudo cumplir su papel de catalizador de la inversión privada. Las transferencias públicas europeas reales -sin contar la ayuda bilateral, sólo la comunitaria- ascendieron a 890 millones de euros, la cuarta parte de lo previsto, como se analizará en detalle.

En consecuencia, el nivel absoluto de bienestar no experimentó cambios cualitativos, especialmente teniendo en cuenta la evolución poblacional. En efecto, el crecimiento demográfico en el área era y es exponencial, y aún más lo es el crecimiento de la población activa. Según previsiones del Banco Mundial, la población de los ocho países más poblados y menos ricos del Sur (Marruecos, Túnez, Argelia, Egipto, Siria, Jordania, Líbano y Turquía) pasará de 213 millones de habitantes en 1997 a 283 millones en 2015, y la fuerza laboral, de 133 millones, a 209 millones. 
El Mediterráneo, más cerca y más lejos

CUADRo 2. Inversión extrajera directa.

Distribución entre regiones emergentes

\begin{tabular}{|l|c|c|c|}
\hline & $1990-94$ & $1995-98$ & Desviación (\%) \\
\hline Total países en desarrollo & 33,8 & 33,2 & $-4,4$ \\
Latinoamérica y Caribe & 10,5 & 12,2 & 16,7 \\
Sudeste Asiático (excluida China) & 10,3 & 8,1 & $-21,2$ \\
Europa central y oriental & 2,4 & 3,5 & 44,1 \\
Región mediterránea (a) & 1,2 & 0,7 & $-37,9$ \\
\hline
\end{tabular}

(a) incluye solamente los países pobres de la ribera sur, excluida Palestina: Marruecos, Túnez, Argelia, Egipto, Jordania, Líbano, Siria y Turquía.

Fuente: Naciones Unidas, Informe Inversiones mundiales.

Si se tiene en cuenta que el crecimiento de la población y el de la población activa aumentaron a un ritmo del $2,3 \%$ y del $2,9 \%$, respectivamente, entre 1980 y 1997, todo indica -expresado sea con cautelas, dada la falta de armonización temporal de las cifras oficiales citadas- que el aumento demográfico absorbió la mayor parte del crecimiento de la riqueza global, cifrado en el 4,1 para el segundo quinquenio de los noventa. Más armonizada temporalmente es la cuantificación del cuadro número 3.

Del mismo se deriva que la población del sur euromediterráneo creció en el sexenio un $9,89 \%$, un ritmo superior a tres puntos respecto de la población mundial (6,8\%), de la que en 1995 representaba el 3,79\%, y en 2000 , el $3,91 \%$. Por consiguiente, en torno a un punto y medio del crecimiento del PIB (del 4,1\% anual en el cuatrienio 1996-1999), quedó absorbido por la explosión demográfica.

Es decir, todo indica que en el mejor de los casos la riqueza per cápita aumentó sólo muy levemente en términos absolutos.

Para mayor preocupación, las proyecciones del Banco Mundial indican que la absorción de la población sureña que se incorpora al mercado de trabajo exigiría un crecimiento del $7 \%$, tres puntos más que los registrados (y por ende, la creación de unos 45 millones de empleos a lo largo de una década). Por tanto, la situación global del desempleo necesariamente ha empeorado.

En términos relativos, los que resultan más significativos en relación al bienestar comparado, de fomento objetivo a los flujos migratorios, y de tensión política subyacente, la distancia de riqueza individual entre el 


\section{Xavier Vidal-Folch}

CuADRo 3. La explosión demográfica

\begin{tabular}{||l|c|c||}
\hline & $\begin{array}{c}\text { Población } \\
\mathbf{1 9 9 5}\end{array}$ & $\begin{array}{c}\text { Población } \\
\mathbf{2 0 0 0}\end{array}$ \\
\hline Argelia & 28.600 .000 & 30.291 .000 \\
Marruecos & 27.100 .000 & 29.878 .000 \\
Túnez & 8.896 .000 & 9.459 .000 \\
Egipto & 59.200 .000 & 67.884 .000 \\
Jordania & 5.439 .000 & 4.913 .000 \\
Líbano & 3.009 .000 & 3.496 .000 \\
Siria & 14.310 .000 & 16.189 .000 \\
Israel & 5.400 .000 & 6.040 .000 \\
Gaza-Jericó ${ }^{*}$ ) & 793.000 & 1.077 .000 \\
Turquía & 61.900 .000 & 66.700 .000 \\
Chipre & 742.000 & 784.000 \\
Malta & 366.000 & 390.000 \\
TOTAL & 215.755 .000 & 237.101 .000 \\
POBLACION MUNDIAL & 5.666 .000 .000 & 6.055 .000 .000 \\
\hline
\end{tabular}

(*) El dato de la columna del año 2.000 es el de 1999.

Fuente: «El Estado del mundo», ediciones de 1996 y de 2002, Akal, Madrid.

Norte y el Sur se ha venido incrementando, según revela el cuadro número 4:

Probablemente el Mediterráneo era y es la región del mundo en la que coexisten, dentro de un espacio más pequeño, las mayores disparidades de desarrollo económico. Una fotografía más sintética: un tercio de la población sigue viviendo bajo el umbral de pobreza, cifrado en dos dólares diarios per cápita. Y el analfabetismo sigue superando el $50 \%$ de la población.

En ese negativo panorama, destaca un territorio en que la situación es más sangrante, Palestina. Sólo dos años después de la Conferencia de Barcelona, el balance de Bruselas sobre lo obtenido en cinco años resultaba así de explícito ${ }^{9}$ : El «enorme esfuerzo» de cooperación financiera realizada «ha fracasado» en el objetivo, aunque (entonces) no en el de «mantener vivo el proceso de paz».

En ese período, en el que los Quince invirtieron más de 1.600 millones de euros en distintos conceptos, el PIB palestino se redujo en un $36 \%$; 
El Mediterráneo, más cerca y más lejos

CUADRO 4. Riqueza relativa Norte/Sur $\left(^{*}\right)$

\begin{tabular}{|l|r|r|r|}
\hline & 1990 & 1999 & 2001 (**) \\
\hline Media comunitaria & 100,0 & 100,0 & 100,0 \\
Media del Sur $(* * *)$ & 20,8 & 19,9 & 17,6 \\
\hline
\end{tabular}

Fuente: Banco Mundial y FMI

(*) Indice de ingresos per cápita medido en paridades de poder de compra ajustadas.

$(* *)$ Estimación

${ }^{* * *)}$ Países contabilizados: Marruecos, Túnez, Argelia, Egipto, Jordania, Siria, Líbano y Turquía

las inversiones privadas se redujeron a menos de la cuarta parte sobre el nivel de 1993; y el desempleo se duplicó, desde el $20 \%$ al $42 \%$ de la población activa. "La principal causa» del fiasco, concluía la Comisión, eran «las medidas adoptadas por Israel para aislar a los territorios»: sólo los cierres de fronteras produjeron unas pérdidas anuales equivalentes al $7,4 \%$ del PIB palestino.

Los únicos dividendos sensibles arrojados por la cooperación europea en Gaza y Cisjordania, aparte de una importante mejora de las infraestructuras -puerto y aeropuerto-, se situaban en el terreno político: «ha evitado el hundimiento de la Autoridad Palestina» $\mathrm{y}$ "ha mantenido vivo el proceso de paz».

\section{Las causas del fiasco}

Por supuesto que la causa principal de esos magros logros radicaba en el agravamiento del conflicto de Oriente Próximo. En 1995, con Rabin y Peres, parecía apuntar a su encauzamiento y desde entonces casi todo han sido malas noticias. Este conflicto contaminó y secuestró el proceso euromediterráneo, pero no fue el único responsable de sus desiguales logros.

Otra causa, frecuentemente olvidada, procede de la dispersión y la lentitud reformista en el Sur. «Da Oriente a Occidente in ogni punto è divisione», decía Leonardo $\mathrm{Da}$ Vinci del Mediterráneo, un retrato que rige hoy para los sureños: divisiones políticas entre dictaduras férreas, democracias imperfectas y sistemas en transición; dispersión ideológica entre fundamentalistas y regímenes moderados; distancia entre países aptos para incorporarse de inmediato a la moneda única europea (Chipre) y otros subdesarrollados y aplastados (Palestina).

La lentitud en las reformas económico-administrativas, especialmente si se compara su ritmo con otra zona emergente, Latinoamérica, en lo 


\section{Xavier Vidal-Folch}

que se refiere a las privatizaciones, la creación de un marco de seguridad jurídica, y el estímulo técnico al proceso de integración regional -a lo que se une la persistencia de unas economías frecuentemente oligopolizadas en torno a un escaso número de familias estrechamente vinculadas al poder político-, constituyen un paisaje que perjudica la atracción de capitales y ralentiza el desarrrollo.

El problema radica, según la Comisión Europea, en «la diversidad de normas, la ausencia de acumulación de normas de origen, una infraestructura física y administrativa insuficiente y la falta de un marco jurídico y económico transparente para la actividad empresarial» y en que en algunos países «las empresas públicas se han opuesto a la reestructuración necesaria para competir en mercados más abiertos, y otros grupos de interés han hecho que se estancaran reformas económicas más amplias» ${ }^{10}$.

Un tercer factor explicativo del desigual resultado del proceso es el escaso nivel de la solidaridad real exhibida por los socios del Norte. Las transferencias financieras directas de Europa a la ribera sur han sido inferiores no sólo a lo necesario sino también a lo programado, como veremos. Y se corre el peligro de que esta distancia aumente en el futuro inmediato. El paquete de perspectivas financieras plurianuales aprobado por los Quince en la cumbre de Berlín (1999) para el primer septenio del siglo XXI redujo sensiblemente la línea presupuestaria dedicada a financiar la política exterior, desde los 5.908 millones de euros a 4.580 millones, perdiendo así casi una cuarta parte (el 22,4\%) de sus recursos. Si bien hay que matizar que esta pérdida se compensa, al menos parcialmente, porque el apoyo financiero a los candidatos de la Europa central y del Este dejará de ser durante el período «ayuda exterior» para convertirse en política interna, estructural y agrícola; y porque se han registrado posteriores correcciones durante los procedimientos presupuestarios anuales.

En cualquier caso, el paralelismo fundacional -cifrado al céntimo en la cumbre de Cannes- entre el Este y el Sur no ha podido mantenerse en la práctica. El surgimiento súbito de otras prioridades, como la rehabilitación de Kosovo y otras acciones en los Balcanes, con su correlato de regateo de fondos comunitarios, y el mantenimiento de una cierta distancia política entre los Estados miembros más distantes geográficamente del Mediterráneo, han contribuido a la caída del Sur de la lista de primeras prioridades a la que había sido trabajosamente aupado.

Así, la intensidad de la ayuda per cápita de la UE al área euromediterránea es la más baja de cuantas se dispensan a las regiones vecinas con las que mantiene vínculos preferenciales, incluso después de las me- 
joras que -como veremos- se registraron en este capítulo desde el año 2000. No alcanza siquiera a la cuarta parte de la vehiculada a los países de la Europa central y oriental, como indica el cuadro número 5:

CuAdro 5. Ayuda financiera de la UE

\begin{tabular}{|l|c|}
\hline & $\begin{array}{c}\text { Créditos de pago y de } \\
\text { compromiso pendientes, 2001 }\end{array}$ \\
\hline Regiones & 83,7 \\
- Balcanes orientales 83,7 & \\
- Países candidatos a la adhesión & 77,6 \\
(excluidos los mediterráneos) 77,6 & 19,0 \\
- Sur mediterráneo 19,0 & 19,7 \\
- ACP (Africa, Caribe, Pacífico) 19,7 & \\
\hline
\end{tabular}

Cifras en euros per cápita

Fuente: Comisión Europea y Banco Mundial

A esta baja intensidad de la ayuda contribuyen sin duda los problemas de ejecución de los presupuestos comprometidos, que situaron el gasto real en una porción mínima del previsto, como indica el cuadro número 6:

A final del ejercicio 1999, por tanto, la divergencia acumulada entre el total de recursos comprometidos y el total realmente hecho efectivo ascendía a 2.545 millones de euros. O lo que es lo mismo, el nivel de ejecución sobre el techo de gasto teóricamente disponible fue sólo del $26 \%$, en promedio para todo el período. $\mathrm{O}$ sea, prácticamente tres cuartas partes de los recursos previstos quedaron sin movilizar.

¿Motivos de esta ineficacia? Por un lado, el atraso de las administraciones sureñas, incapaces en algunos casos de absorber las ayudas, como lo demuestra que en el primer quinquenio países tan significativos como Turquía (o Siria, aunque ésta no había firmado aún la asociación) fuesen incapaces de presentar un solo proyecto.

Por otro, los cuellos de botella europeos. El comisario Chris Patten resumía así los dos tipos de problemas existentes: «Por un lado están los largos procedimientos burocráticos para la autorización de los gastos, pero por otro el gran problema es que el Consejo de ministros autoriza partidas de gasto, pero luego no respalda los medios presupuestarios para que la Comisión pueda gestionar ese gasto» ${ }^{11}$, es decir, que la maquinaria burocrática estaba infradotada, el personal era escaso y no al- 
CUADRo 6. Ayudas EUROMED ejecutadas/ayudas comprometidas

\begin{tabular}{|l|c|c|c|c|c|c|}
\hline & 1995 & 1996 & 1997 & 1998 & 1999 & Total \\
\hline $\begin{array}{l}\text { Créditos de pago } \\
\text { (Ayudas ejecutadas) }\end{array}$ & 50 & 155 & 211 & 231 & 243 & 890 \\
$\begin{array}{l}\text { Créditos de compromiso } \\
\text { (Ayudas comprometidas) } \\
\text { Cociente pagos/compromiso }\end{array}$ & 173 & 403 & 981 & 941 & 937 & 3.435 \\
\hline
\end{tabular}

En millones de euros. Este cuadro recoge únicamente la ayuda presupuestaria directa, a la que habría que añadir la asistencia financiera a través de los préstamos del Banco Europeo de Inversiones, cuyo monto total previsto para el período ascendía a 4.672 millones de euros.

Fuente: Comisión Europea

canzaba a vehicular, con las garantías requeridas, los techos autorizados de gasto.

De manera que al ritmo de aquel momento, alertaba, la ayuda total comprometida con los países mediterráneos después de 1996 tardaría 8,75 años en completarse, por siete años en el caso de Asia y 6,5 en el de Latinoamérica. Los Balcanes constituían la excepción, 2,52 años. Una incapacidad de severo impacto, puesto que tiene como efecto difuminar el relevante hecho de que la UE concede el $55 \%$ de toda la ayuda internacional al desarrollo y un $66 \%$ de toda la ayuda general (incluyendo la ayuda humanitaria de urgencia).

La puesta en marcha de los programas de cooperación fue difícil. El reglamento Meda-1, que vehiculaba la ayuda financiera, se atrasó hasta julio de 1996, ocho meses, con lo que durante la fase de arranque no se podían efectuar pagos por falta de base jurídica. La Unión vivió enseguida el drama de las «vacas locas», que provocó una parálisis política y una búsqueda de recursos con que financiarla.

Y la recién nacida Comisión Santer se vio abocada a la crisis que desembocaría en su dimisión, desencadenada precisamente por la gestión de los programas Med, los precedentes del Meda. Las irregularidades descubiertas por el Tribunal de Cuentas eran relativamente menores (2,2 millones de euros) y la Comisión los paralizó inmediatamente. Pero sirvieron para enhebrar el acoso al Ejecutivo comunitario, situar al horizonte marenostrum bajo sospecha de derechistas y calvinistas interesados, inhibir a los funcionarios y ralentizar el nuevo proceso euromediterráneo. El verdadero escándalo radicaba en la asimetría: un cúmulo de irregularidades de muy superior calado, cifradas por el mismo tribunal 


\section{El Mediterráneo, más cerca y más lejos}

en 610 millones de euros (en el programa TACIS, de ayuda a Rusia) apenas tuvieron consecuencias... Pero es que Rusia es Este, no Sur.

Junto a estos obstáculos de intendencia figuraron otros más de fondo, derivados de la propensión al proteccionismo agrícola de los Estados miembros de la UE ${ }^{12}$, que dificultó y frenó la negociación de casi todos los Acuerdos de Asociación, por la cortoplacista defensa del tomate nacional (España contra Marruecos), de la flor cortada (Holanda) o del arroz (frente a Egipto). En todos ellos los Gobiernos tendieron a erigirse en correas de transmisión de los respectivos lobbies agrícolas -sustentados en un porcentaje mínimo de sus poblaciones, -pero dotados de una gran capacidad de movilización pública y de influencia mediática- para emprender forcejeos numantinos.

La gravedad de esta miopía europea bien merece ejemplos y detalle de responsabilidades. Así, en el caso del acuerdo con Marruecos, el sector tomatero dio la batalla en el último bimestre de 1995 contra un aumento de las exportaciones alauitas cifrado en 15.000 toneladas anuales. La nimiedad de la concesión se constata al recordar que en los anteriores cuatro años las exportaciones españolas de ese producto a la Comunidad se habían duplicado, hasta alcanzar las 700.000 toneladas. De manera que, en el peor de los casos, los agricultores españoles se veían abocados a sacrificar en beneficio de sus colegas del otro lado del estrecho jel $0,042 \%$ de sus progresos comercialesi. Peor aún. La última resistencia vino de la tripleta Holanda-Alemania-Bélgica, que se negaba a unas concesiones para la exportación de flor cortada marroquí, cifradas en menos de 20 millones de euros, ignorando que Rabat había dado ya entrada a cereales, leche y otras producciones propias del Norte europeo. ¡Todo ello en un marco en el que las exportaciones agrícolas de la UE al Sur superan a las de sentido inversoj.

Por lo demás, la afluencia de oleadas migratorias, desde el Magreb a España, desde los Balcanes y Turquía hacia Italia y Alemania, han consolidado los recelos ancestrales de los europeos y la percepción hegemónicamente securitaria de las relaciones Norte-Sur. «El telón de acero, institucional y militar, ha dejado paso a otro telón de acero, mental y político. El primero, que ahora ha caído, se encontraba en el Este, iy el segundo se erige acto seguido frente al Suri», escribía hace diez años Bichara Khader ${ }^{13}$. «De hecho, el racismo antimagrebí, antimusulmán, antiárabe, ha venido a reemplazar al anticomunismo, al antisovietismo, en términos de reorientación de los proyectos conservadores. La noción de extranjero se ha modificado. El hombre del Este, que durante mucho tiempo había sido considerado extranjero y estigmatizado por media humanidad, es redescubierto ahora como alguien cercano, como un hermano. El extranjero ha pasado a ser el hombre 


\section{Xavier Vidal-Folch}

del Sur», concluía. Nada sustancial modificó en positivo esa percepción. Y el resultado es que proyectos como el del Estatuto del Residente, empujado por el comisario António Vitorino para homogeneizar al menos el estatuto legal de los cerca de 16 millones de inmigrantes instalados en los distintos Estados miembros de la UE, sufría retraso tras retraso.

\section{Examen de conciencia: desde Marsella}

De modo que cinco años después de iniciado, el proceso euromediterráneo exhibía notables déficits en sus procedimientos y en sus resultados. La celebración del primer quinquenio sirvió para efectuar examen de conciencia y propósito de la enmienda. Desde, sobre todo, la cumbre de Marsella (15 y 16 de noviembre de 2000) los Quince trataron de revitalizarlo, con nuevos programas y diversos ajustes administrativos y procesales de la maquinaria comunitaria.

Este impulso político - del que Francia se erigió en principal protagonista, por delante de España, y con Italia en los márgenes- logró en poco menos de dos años algunos resultados tangibles.

Así, de los cinco acuerdos de asociación pendientes, desde Marsella se avanzó en tres, al firmarse el de Egipto y concluir las negociaciones con Argelia y Líbano (final de 2001). Pero la ratificación de la asociación con Jordania seguía pendiente y la negociación con Siria, empantanada. Pese a ello, la trama de acuerdos establecidos -y la posibilidad de la entrada en vigor provisional y anticipada de algunas de sus cláusulas- diseñaban ya una «masa crítica» suficiente.

También, a raíz de la modificación del reglamento Meda en 2000, se descentralizó la gestión del gasto público y se concentró la maquinaria administrativa de Bruselas, lo que redundó en mejoras en las transferencias financieras, duplicándose en 2001 la ratio de ejecución del gasto público, como se aprecia en el cuadro número 7.

¿Hubo Mejoras en la transferencia absoluta de recursos efectivos pagados (de 243 millones de euros en 1999 a 402 millones en 2001) y de gestión en términos relativos (de ejecutarse el $26 \%$ de los compromisos en 1999, hasta el 53\% en 2001)? Cierto; pero ésta última ambivalente, porque decreció el techo de los créditos de compromiso (de 937 millones de euros en 1999 a 756 millones en 2001). ¿Por qué? Porque se eliminaron los compromisos antiguos, anteriores a 1995 no ejecutados y los compromisos «en hibernación» (aquellos por los que no se ha hecho ningún pago en dos ejercicios consecutivos), con lo que se redujeron 310 millones de euros al final de 2001. Es decir, se gestionó mejor y aumentó la coopera- 
El Mediterráneo, más cerca y más lejos

CUADRo 7. Ayudas EUROMED ejecutadas/ayudas comprometidas

\begin{tabular}{|l|r|r|r|r|r|r|r|r|}
\hline & 1995 & 1996 & 1997 & 1998 & 1999 & 2000 & 2001 & Total \\
\hline $\begin{array}{l}\text { Créditos de pago } \\
\text { (Ayudas ejecutadas) }\end{array}$ & 50 & 155 & 211 & 231 & 243 & 330 & 402 & 1.622 \\
$\begin{array}{l}\text { Créditos de compromiso } \\
\text { (Ayudas comprometidas) }\end{array}$ & 173 & 403 & 981 & 941 & 937 & 879 & 756 & 5.071 \\
Cociente pagos/compromiso & $29 \%$ & $38 \%$ & $21 \%$ & $24 \%$ & $26 \%$ & $37 \%$ & $53 \%$ & $32 \%$ \\
\hline
\end{tabular}

En millones de euros.

Fuente: Comisión Europea.

ción efectiva, pero se aparcó la ambición de mantener las previsiones del paralelismo entre el Sur y el Este. Hubo mejoras también en la financiación del desarrollo, con la creación de un nuevo instrumento de capitalriesgo gestionado por el BEI.

Y se registraron avances en distintos ámbitos de la colaboración civil: la Comisión lanzó más de 400 proyectos regionales sobre asuntos como la conservación del patrimonio, el sector audiovisual y la juventud. Mejoró la disposición de los países extracomunitarios a incrementar la apertura recíproca de sus mercados, como apuntó el compromiso de establecer una subzona de libre comercio Sur-Sur, adoptado en Agadir en 2001. Y aumentó la densidad de los planteamientos para acelerar la liberalización de los intercambios, especialmente en las reuniones de los ministros de Comercio.

Así, los Quince acordaron reexaminar el ritmo de la liberalización agrícola, factor crucial para el reequilibrio de las relaciones comerciales, como respuesta a las demandas del Sur y a las evidencias ofrecidas por nuevos estudios económicos. Según uno de éstos, en el supuesto de apertura de los mercados agrícolas europeos, las exportaciones agrícolas del Sur aumentarían en un $27 \%$ en cinco años, flujo que equivaldría a la entrada de un volumen de productos equivalente al $11 \%$ de los actuales intercambios intracomunitarios, aunque alcanzarían el $75 \%$ en el caso de las frutas ${ }^{14}$.

También se abrió paso el compromiso para incorporar a los sureños al sistema paneuropeo de acumulación de origen, cuya aplicación debería multiplicar el nivel de comercio efectivo. «La evidencia indica», según un estudio académico, «que el comercio agregado entre países carentes de 


\section{Xavier Vidal-Folch}

acumulación es de aproximadamente el $40 \%$ de lo que sería en caso contrario» ${ }^{15}$.

Otras iniciativas, maduradas en vísperas de la convocatoria de la cumbre de Valencia, debían complementar las anteriores iniciativas. Así, la extensión del programa TEMPUS de intercambios entre universitarios -ya existente con los Balcanes y los países de la antigua URSS,extensión financiada con más de veinte millones de euros anuales. Así, la propuesta de crear una Fundación euromediterránea para fomentar el diálogo entre culturas, que se dotaría con un millón de euros aportado por cada uno de los Quince y por la Comisión, y contribuciones voluntarias de los socios sureños. Así, la creación de una línea especial dentro del BEI, como sucedáneo del Banco Euromediterráneo sobre el que los Quince no alcanzaron consenso durante el Consejo Europeo de Barcelona (marzo de 2002), que debía compensar el escaso desarrollo de la intermediación financiera en el Sur y su falta de eficiencia y competencia.

El balance del primer septenio del proceso, por tanto, sin cubrir ni de lejos las expectativas suscitadas en su inicio, mejoró respecto al del primer quinquenio.

Quizá convenga ahora establecer alguna comparación. Si la arquitectura de la región euromediterránea era de mejor y más completa factura que la norteamericana del TLC (1993/1994), quizá no pueda decirse lo mismo de su puesta en práctica. Es verdad que el TLC contemplaba formas mínimas de integración, al prever sólo la libre circulación de bienes y servicios (no de los factores de producción) y la unión aduanera (al conservar cada socio su propia política comercial).

Y también lo es que la ausencia de una estrategia de cohesión ex ante mediante ayudas al desarrollo del socio menos próspero no evitó que cuando - por la crisis social de Chiapas y el asesinato del candidato presidencial Ernesto Colosio, pero, no se olvide, simultáneamente a la entrada en vigor del TLC- en 1994/1995 México atravesó la gran crisis financiera conocida como «el tequilazo», EEUU tuviera que acudir, esta vez ex post, aportando ingente ayuda financiera: 20.000 millones de dólares.

Pero, a cambio, el compromiso de recíproca liberalización comercial alcanzaba todos los sectores. En el ámbito agrícola, el acuerdo implicaba eliminar tarifas, cuotas de importación y licencias, si bien de forma gradual, en 15 años, con carácter absoluto. Las rebajas arancelarias han sido continuadas (en 1997, 1998, 2000 y enero de 2002) y el intercambio comercial de México con EEUU se ha triplicado por tres, hasta superar los 200.000 millones de dólares, lo que desbancó a Japón como segundo 
socio comercial de EEUU, detrás de Canadá. De esta forma el Sur-México ha empezado a lograr un mejor acceso a los mercados norteamericanos, ha espoleado su producción -también en sus «maquiladoras» fronterizas, transformadoras o ensambladoras de producciones semielaboradas importadas- y ha atraído capitales, incrementando su empleo, lo que no ha reducido los flujos de emigración al Norte, pero los ha hecho más manejables.

Ultima, pero no menor, consideración. Como todo acuerdo regional implica un efecto de desviación de comercio, la participación de la UE en el comercio exterior de México se redujo del $11 \%$ en 1990, al 6\% en 1999 , factor que aceleró los trabajos para un nuevo Tratado México-UE, con la declarada intención de esta última de recuperar el terreno perdido.

Cierto que no es lo mismo negociar y aplicar un acuerdo entre tres países (EEUU, Canadá y México) que entre un bloque semiintegrado de 15 y una retahíla desintegrada de 12 .Y que es mucho más complejo un acuerdo de asociación que otro de mera liberalización comercial. Pero esa realidad no oscurece la diferencia de balances.

\section{Desde el 11 de sepiembre}

De modo que, con todas sus limitaciones, Europa y sus socios sureños lograron relanzar desde el año 2000 su proyecto común. Pero no es tan seguro que hayan extraído todas las consecuencias que para éste supone el nuevo escenario mundial dibujado tras los atentados terroristas del 11 de septiembre de 2001.

En efecto, han surgido nuevos datos aún pendientes de elaboración política. Como el hecho de que la asfixia del pueblo palestino haya sido manipulada, tratando de convertirla en coartada ideológica, por el terrorismo ultraislamista (Bin Laden). Como la evidencia de la labor preparatoria desarrollada en Europa -de Alicante a Hamburgo- de aquellas acciones, a través de densas y sutiles redes. Como el hecho de que de la lista de los 22 terroristas más buscados por el FBI hecha pública el 10 de octubre de 2001 por el presidente norteamericano, George W. Bush, once, esto es, exactamente la mitad, sean originarios de distintos países ribereños. O como la reverdecida y creciente sensibilidad de las opiniones públicas del Norte de Africa ante la evolución de la política israelí sobre la cuestión palestina, especialmente desde la guerra desencadenada contra Yasir Arafat en la pasada Semana Santa.

Novedades de distinta factura como las reseñadas podrían resituar al Mediterráneo como escenario de una de las principales líneas de fractu- 
ra del pretendido «choque de civilizaciones» que auguró Samuel Huntington. Las críticas al exceso de culturalismo que atrajo su planteamiento y la evidencia de que la dialéctica Norte/Sur sigue mostrando su vigor incluso en el interior del nuevo mapamundi que propone, ¿autorizan a obviar que el diálogo entre civilizaciones se ha erigido en una de las principales prioridades para los distintos actores del entorno mediterráneo?

Desde septiembre de 2001 algunas de las cosas sabidas pero que provocaban miradas huidizas cobran nueva importancia. En la zona mediterránea existe una innombrada potencia nuclear y un par de potencias armamentísticas, químicas o bacteriológicas, lo que complica el capítulo de seguridad del proceso de agrupación regional. La técnica utilizada hasta ahora, consistente en obviar fenómenos tan fundamentales, ha llegado probablemente a su límite de eficacia, y convendrá abordar estos asuntos de manera frontal.

Además, el traspaso del terrorismo internacional desde la agenda exterior de los EEUU a la de la seguridad interior ha servido como acicate para realinear los intereses nacionales de los distintos Estados en función de ese fenómeno, y como excusa ocasional para estrategias políticas que hace un decenio hubieran resultado impresentables. Así sucede, desde luego, con el conflicto checheno, para Rusia; pero también con el del Polisario, para Marruecos; el kurdo, para Turquía; o la Kabilia y la minoría bereber para Argelia.

Pese a todo, es difícil hallar en el mundo otras tan extensas zonas de frontera que hayan puesto en marcha, aún con las limitaciones del caso, procesos de integración como el abierto por la Declaración de Barcelona. A punto de amortizarse la línea del telón de acero que dividió al viejo continente durante medio siglo -gracias al proyecto de ampliación de la UE-, seguramente solo queda ésa.

Europa es protagonista esencial del proceso. Y sólo si juega con decisión todas sus cartas será capaz de extraer todas las posibilidades del diseño euromediterráneo.

Pero jugar todas las cartas exige eso, que sean todas, no sólo la administrativa, la económica o la comercial, sino también la de política exterior y en su caso, la defensa. Y si, aunque sean escenarios diferentes, los del Euromed y del Próximo Oriente se superponen, hasta el punto que una de las principales causas de los magros resultados de aquél hay que encontrarlas en éste, habrá que concluir en que la acción exterior de la UE sobre el conflicto israelo-palestino ha sido hasta el momento insuficiente.

Es cierto que en los últimos años la Unión ha incrementado su protagonismo en el área. Constituyen jalones de esa dinámica el nombra- 
miento (1999) de un enviado especial permanente, Miguel Angel Moratinos, a Jerusalén; la actividad desplegada por el Alto Representante para la Política Exterior y de Seguridad Común, Javier Solana (con su presencia en la cumbre de Sharm el Sheik o su participación en el Comité Mitchell); o la continua influencia sobre la política exterior norteamericana, principal potencia y la única capaz, como generalmente se reconoce, para liderar la reconducción del proceso de paz. Estos esfuerzos han desembocado en un resultado, la pervivencia -cada vez más amenazada- de una Autoridad Palestina, que sin la cobertura de la Unión -y el lábil apoyo de la Liga Arabe- hace tiempo habría dejado de existir.

Pero cada vez son más las voces que reclaman una actuación más enérgica de la Unión. Tanto hacia la Autoridad Palestina -cuyo funcionamiento democrático y cuyos compromisos de pacificación han exhibido importantes déficit- como, sobre todo, sobre Israel. Es cierto que la fragua de una posición común entre los Quince topa con algunas herencias nacionales difíciles de cohonestar. Y que por distintos motivos, tres Estados miembros suelen ser renuentes a enervar posturas de exigencia: Alemania, a causa de su mala conciencia histórica por el holocausto judío perpetrado en época nazi; Holanda, por la influencia de su lobby proisraelí; y el Reino Unido, por su estrecha alianza estratégica con Washington.

El resultado ha sido una política de muy mínimo común denominador. Y sus consecuencias, dramáticas para la UE no ya en términos de poder sino incluso de prestigio. Así lo evidenciaron las negativas del Gobierno Sharon, el pasado abril, a permitir al presidente de turno del Consejo Europeo, José María Aznar, o a la delegación compuesta por Javier Solana y el ministro de Exteriores español, Josep Piqué, visitar al rais asediado en Ramala, mientras pudo hacerlo el enviado del presidente norteamericano.

En consecuencia, pese a las diferentes ópticas existentes entre los Quince, parecía ineludible a ojos de muchos una actuación más exigente sobre Tel Aviv. Sin implicar necesariamente una estrategia de ruptura diplomática ${ }^{16}$, la panoplia de medidas eficaces a disposición es amplia: desde la más dura de una suspensión del acuerdo científico vigente ${ }^{17}$, al adelantamiento de la convocatoria del Consejo de Asociación ${ }^{18}$, o a la simple exigencia de cumplimiento del Tratado de Asociación Euromediterráneo ${ }^{19}$ en lo relativo al fin de los abusos en la exportación de productos palestinos como originarios propios.

Sólo si Europa se respeta a sí misma, esto, sólo si actúa coherentemente con su declarada voluntad de influencia, logrará el respeto pro- 
fundo de los otros protagonistas. Sólo así logrará que los vecinos del Sur la contemplen no únicamente como su primer socio económico sino también como su principal aliado estratégico. Todo ello sin contar con que el relanzamiento de la ribera sur mediterránea resultará imprescindible cabeza de puente para la rehabilitación del continente africano en su conjunto.

\section{Notas}

1 Marruecos, Argelia, Túnez, Egipto, Siria, Jordania, Líbano, Israel, Palestina, Turquía, Chipre y Malta, con Libia de observador).

2 Comunicación de la Comisión «Reforzar la política mediterránea de la UE mediante una asociación euromediterránea", 19/10/1994)

3 «Le pari euro-mediterranéen", Eberhard Rhein en el libro colectivo «Euromediterranée, une région à construire», Publisud, Paris, 1995)

4 COM 1998-538 final, 23/09/1998)

5 Los países que se apuntarían posteriormente serían Argelia, Libia, Mauritania, Siria y Palestina. Un antecedente de esta zona de libre mercado fue la Unión del Magreb Arabe, UMA, lanzada en los primeros años noventa, que no logró cristalizar a causa del enfrentamiento argelino-marroquí, la guerra civil argelina y el embargo internacional a Libia.

6 Comunicación de la Comisión: "Un nuevo impulso para el proceso de Barcelona", 6/9/2000, COM 2000-497 final).

7 Comunicación de la Comisión «Sobre la Asociación Euromediterránea y el mercado único, 1998).

8 EL PAIS, 24/11/1995).

9 Comunicación de la Comisión: «El papel de la UE en el proceso de paz y en la asistencia futura al Próximo Orientem" 14/01/1998).

10 COM 2000-497).

11 EL PAIS, 8/08/2000).

12 Hablamos específicamente de proteccionismo agrícola y no de otro. Es falsa la percepción, difundida sobre todo por EEUU al menos desde el Acta Unica de 1995 que dio paso al Mercado Interior, según la cual Europa es la potencia más cerrada del mundo. $\mathrm{Al}$ revés, es de las más abiertas. En 2000, Europa occidental absorbía el 39,6\% de las importaciones mundiales de mercancías, contra el 23,2\% de EEUU y el 5,8\% de Japón, según el informe estadístico de la OMC, edición 2001. Y ciñéndonos a la UE, excluidas las importaciones intracomunitarias, EEUU supuso el 23,9\%; la UE, el 18,3\%; y Japón, el $7,2 \%$. Ocurre que el proteccionismo europeo se concentra en un sector muy sensible para los países en desarrollo, la producción agrícola. También practica ese proteccionismo EEUU, aunque aparece formalmente más diluido al plasmarse en la subvención de las rentas a los agricultores, más que en el subsidio público a los precios. Las sucesivas reformas de la Política Agrícola Común, también empujadas por la filosofía de la OMC, tienden a reducir la política de precios en favor de las ayudas directas al agricultor y al desarrollo rural).

13 Bichara Khader, «Europa y el Gran Magreb», Fundación Paulino Torras Doménech, Barcelona (1992). 


\section{El Mediterráneo, más cerca y más lejos}

14 «El impacto de la liberalización comercial euromediterránea en los intercambios agrícolas", resumen ejecutivo del estudio elaborado para FEMISE, A. Lorca y J. Vicens (UAM, 2000).

15 «Estudio sobre el impacto económico de la extensión del sistema paneuropeo de acumulación de origen a los socios mediterráneos del proceso de Barcelona", Informe intermedio, Instituto Europeo de la Universidad de Sussex (Patricia Augier, David Evans y otros).

16 Una medida inmediatamente menos dura sería el envío de una comisión europea para investigar las eventuales violaciones de los derechos humanos, algo que permite la cláusula democrática del "Acuerdo Euromediterráneo por el que se crea una asociación entre las Comunidades Europeas y sus Estados miembros, por una parte, y el Estado de Israel, por otra" (Diario Oficial 21/06/2000), que en su artículo 2 establece: "Las relaciones entre las Partes, así como todas las disposiciones del presente Acuerdo, se fundamentan en el respeto de los principios democráticos y de los derechos humanos, que inspira sus políticas interiores y exteriores y constituyen un elemento esencial del presente Acuerdo". Una medida más contundente sería la exigencia en los foros internacionales, por los Estados miembros, de reparaciones por los daños causados por el Ejército israelí a los proyectos financiados por los Quince. La medida más dura sería la denuncia del Acuerdo en todos sus aspectos, de diálogo político, comerciales y sociales, según su artículo 82: «El presente Acuerdo se celebra por tiempo indefinido. Cualquiera de las Partes podrá denunciar el presente Acuerdo mediante notificación a la otra Parte. El presente Acuerdo dejará detener efecto seis meses después de la fecha de dicha notificación».

17 Suspensión o cancelación del "Acuerdo de Cooperación científica y técnica entre la Comunidad Europea y el Estado de Israel», Diario Oficial 27/03/1999. Otorga a Israel un estatuto prácticamente equivalente al de los Estados miembros como copartícipe del quinto programa marco de investigación. Toma parte en su Consejo, contribuye con su cuota y es beneficiario, junto a los miembros de la UE y del Espacio Económico Europeo, de sus programas. En general, los países que obtienen más "retorno" nacional de la política científica son los tecnológicamente más avanzados. El interés de Israel es doble: extrae buen rendimiento de su propia capacidad tecnológica (aplicaciones civiles de tecnología de Defensa), y calibra la evolución de sus socios. El artículo 12 del acuerdo reza: «Cualquiera de las Partes contratantes podrá poner término al presente Acuerdo en cualquier momento, con un preaviso de doce meses».

18 Convocatoria del Consejo de Asociación. Este Consejo es el organismo encargado de la gestión del Acuerdo de asociación. De composición bilateral, se reúne anualmente "y cada vez que sea necesario" (artículo 67 y siguientes), adoptando sus decisiones mediante consenso. En caso de conflicto, cada Parte nombra un árbitro y los dos árbitros, a un tercero; la junta de los tres árbitrros adopta sus decisiones por mayoría. "Si una de las Partes considera que la otra Parte no ha cumplido alguna de las obligaciones derivadas del presente Acuerdo, podrá tomar las medidas apropiadas", que comunicará al Consejo y sobre las que se abrirán consultas bilaterales si la otra parte lo solicita (artículo 79).

19 El Acuerdo de Asociación establece la liberalización comercial de los productos originarios de Israel y posibilita la «acumulación bilateral» de su origen (productos elaborados en Israel que incorporan algún elemento o material procedente de la Unión), pero «no permite todavía la acumulación diagonal", es decir, la incorporación a los productos israelíes de elementos originarios de otros socios (Comunicación de la Comisión «Aplicación del acuerdo provisional de comercio y asuntos comerciales entre la Comunidad Europea e Israel» (12/05/1998). Según esta comunicación, Israel viola los términos comerciales 
pactados del Acuerdo de Asociación (que entraron en vigor en fecha adelantada a la oficial del 1 de junio de 2001) al exportar como propios los productos provenientes de los asentamientos ilegales en los territorios autónomos y ocupados (anexionados en 1967 y que no forman parte del territorio de Israel, de acuerdo con las resoluciones de NNUU), así como productos elaborados por palestinos de la franja de Gaza y Cisjordania. La Comisión denunció violaciones en el comercio de flores cortadas, zumo de naranja, fresas, berenjenas, cítricos, zapatos y otros productos del cuero, que suponen ingresos fiscales indebidos a la Hacienda israelí -en algunos casos desviados de su destino correcto, la palestina-, dado el trato preferencial que los Quince otorgan a los productos auténticamente originarios. El entonces vicepresidente Manuel Marín denunció estas violaciones en términos muy contundentes. El expediente se empantanó, hasta la reunión del comité de cooperación aduanera celebrada en Jerusalén el 23 y 24 de julio de 2001, en la que Israel defendió su actuación, los Quince sostuvieron su postura, y ambos acordaron celebrar una reunión técnica informal antes de final de octubre de 2001. También previeron que en caso de no encontrar una solución, el asunto pasaría a «la instancia competente prevista por el acuerdo de asociación» (el Consejo de Asociación). Desde entonces el asunto está enterrado. 